\title{
Resolution Enhancement of Optical Surface Plasmon Resonance Sensor Using Metamaterial
}

\author{
Sarika PAL ${ }^{1}$, Y. K. PRAJAPATI ${ }^{*}$, J. P. SAINI ${ }^{3}$, and V. SINGH ${ }^{4}$ \\ ${ }^{1}$ Sarika Pal, Department of Electronics \& Communication Engineering, KIET, Ghaziabad (U.P.), India \\ ${ }^{2}$ Yogendra Kumar Prajapati, Department of Electronics \& Communication Engineering, MNNIT, Allahabad-211004 \\ (U.P.), India \\ ${ }^{3}$ Jai Prakash Saini, Department of Electronics \& Communication Engineering, BIET, Jhansi-284128 (U.P.), India \\ ${ }^{4}$ Vivek Singh, Department of Physics, Faculty of Science, Banaras Hindu University, Varanasi (U.P.), India \\ ${ }^{*}$ Corresponding author: Y. K. PRAJAPATI E-mail: yogendrapra@gmail.com
}

\begin{abstract}
In this paper, we present and discuss a path to extend the enhancement of the resolution of an optical surface plasmon resonance (SPR) sensor. Basically, our approach is to combine bi-metamaterial layers to design the SPR sensor. The calculation shows that the proposed SPR sensor structure has a preference over the conventional SPR sensors and bimetallic SPR sensors since it gives a much sharper reflectance dip and can achieve considerable sensitivity improvement when compared to the recently reported investigations. The effects of the metamaterial permittivity, permeability, and thickness on the reflectance curve are studied. It is also seen that metamaterial layers improve the field of the proposed SPR structure, which may provide a novel tool to significantly enhance the sensitivity and resolution of the sensors.
\end{abstract}

Keywords: Surface plasmon resonance sensor, metamaterials, Fresnel equations, reflectance

Citation: Sarika PAL, Y. K. PRAJAPATI, J. P. SAINI, and V. SINGH, "Resolution Enhancement of Optical Surface Plasmon Resonance Sensor Using Metamaterial, 2015, 5(4): 330-338.

\section{Introduction}

Surface plasmon resonance (SPR) sensors provide label free and real-time analysis of optical property changes near a metal-dielectric interface that supports surface plasmons [1]. Surface plasmons are propagating or localized electromagnetic field oscillations that occur at the interface between two materials given that one of the materials has a real negative dielectric constant [2]. The incident light can cause excitation of the surface plasmons. When the momentum of the surface plasmons matches that of the incident light, the so-called surface plasmon resonance phenomenon takes place. In most sensor applications, the plasmon supporting material is gold or silver as it readily supports plasmon modes. Gold displays a higher shift of the resonance angle to the change in ambient refraction index and is chemically stable also. Silver possesses a higher value of imaginary part of refractive index than the gold by which it shows a narrower resonance curve in comparision to gold. Thus, silver provides a higher signal to noise ratio of SPR chemical sensors, but has a poor chemical stability [3]. SPR sensors are more advantageous than other methods, such as

Received: 3 July 2015 / Revised: 3 September 2015

(C) The Author(s) 2015. This article is published with open access at Springerlink.com

DOI: $10.1007 / \mathrm{s} 13320-015-0269-5$

Article type: Regular 
fluorescent tagging, when a quantitative label-free approach to tracking binding occurrences or optical property shifts over time is needed [4]. Traditionally, SPR is measured using the Kretschmann configuration, with a prism and a thin highly reflecting metal layer deposited upon the prism base [5]. Various configurations in a Kretschmann type device structure have been proposed and explored yet, mainly by designing the layer stack on the hypotenuse face of a prism, which include long range SPR sensors [6, 7], Au-Ag metals film SPR sensor i.e. conventional $\operatorname{SPR}[2,8]$, waveguide coupled plasmon-waveguide SPR sensor [9], etc. Particularly, the use of the surface plasmon wave with other plasmonic or guided modes appears to provide the most effective way of sharpening the SPR curve, thus enhancing the sensing resolution [10]. The resolution of an SPR line width of the SPR reflectivity curve decreases, and a shift in the resonance angle or wavelength in response to the environmental index change increases [11, 12].

In recent years, there has been a dramatic proliferation of research concerned with metamaterial (MM) sensors. The history of the metamaterial began with the research paper of Veselago [13]. The metamaterials can manipulate electromagnetic wave beams in surprising ways and exhibit some exotic electromagnetic properties which are not readily available in nature, such as backward propagation [14], reverse Doppler effect, reverse Vavilov-Cerenkov effect [13], negative refraction $[15,16]$, and diffraction-limit breaking imaging [17]. In addition, metamaterials can exhibit a strong localization and enhancement of fields so that these can be used to actually improve the sensor selectivity of detecting nonlinear substances and to enable detection of extremely small amounts of analytes [18]. Based on these properties, many new or improved applications of metamaterials have been proposed recently. For example, using metamaterials instead of metal parts in the SPR sensor was proposed to enhance the sensing performance [19], and utilizing metamaterials as high frequency sensors was also considered [20].

Recently, K. S. Lee et al. [21] obtained the incident angle and reflectance of the bi-metallic five-layer optical SPR sensor using the bi-metallic concept. The use of a silver $(\mathrm{Ag})$ inner coupling layer between the prism and the dielectric waveguide film was confirmed to have advantages in enhancing the sharpness of SPR curves as well as improving the sensing resolution and dynamic range.

Very recently, Y. K. Prajapati et al. [22] showed that SPR sensors using the metamaterial are better than conventional optical SPR sensors for the fourand five-layer structure. The use of the metamaterial layer is confirmed to have an advantage of increasing the reflectance dip of the optical SPR sensor, thus improving the sensitivity in term of the sensing resolution and dynamic range over the bi-metallic SPR sensor [21].

The purpose of this paper is to illustrate the performance of the metamaterial based sensor. We have investigated a plasmonic stack for the resolution enhancement of an SPR sensor based on a waveguide structure through combining a metal by using the bi-metamaterial concept. Theoretical modeling has been carried out by solving the Fresnel equations for a multilayer stack of the metamaterial $/$ metal/metamaterial layer. SPR sensor reflectivity curves are calculated as a function of the incident angle. The result shows that the metamaterial based sensor possesses a much higher sharpness of the reflectivity curve than traditional sensors. Moreover, it is believed that metamaterial based sensors can have a potential application in biochemical sensing in the future.

The paper organization is as follows: modeling section where descriptions of the modeling and related equations/formulations are presented in Section 2. In Section 3, numerical results will be discussed for validating our suggested formulation for an optimal structure for a desired parameter and 
examining the resolution features of the proposed structure. Finally, the paper ends with a short conclusion given in Section 4.

\section{Theoretical modeling}

A schematic geometry of the proposed SPR sensor is shown in Fig. 1.

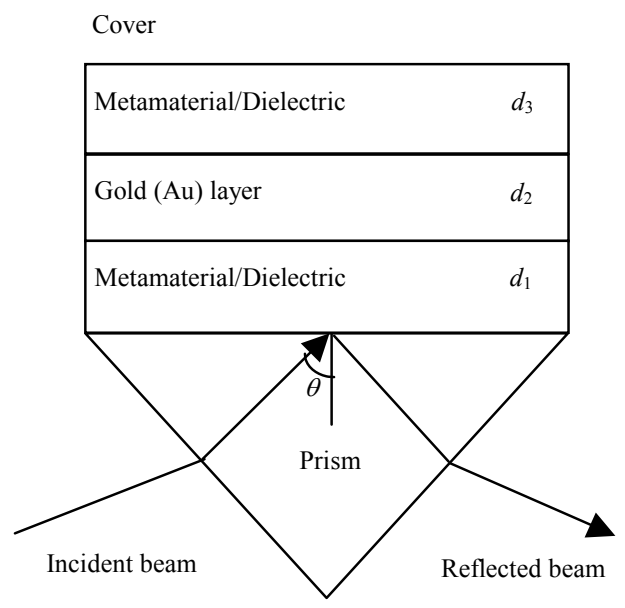

Fig. 1 Proposed optical SPR sensor structure.

It consists of five layers with the bi-metamaterial layer thickness of $d_{1}$ and $d_{3}$, respectively. Table 1 has three different configuration details which are used in this paper. Structure 1 has only a single MM or dielectric layer. Structure 2 is the bi-metallic approach which is the proposed structure. Structure 3 has a thick MM or dielectric layer which is used for the sake of comparison. Table 1 has the corresponding thicknesses of all the structures. The metamaterial layer is characterized by electric permittivity $\left(\varepsilon_{m}\right)$ and magnetic permeability $\left(\mu_{m}\right)$. Park et al. [23] introduced the frequency dependent complex permittivity and permeability in the form

$$
\begin{gathered}
\varepsilon_{m}(\omega)=1-\frac{\omega_{p}^{2}}{\omega^{2}+\mathrm{i} \gamma \omega} \\
\mu_{m}=1-\frac{F \omega^{2}}{\omega^{2}-\omega_{0}^{2}+\mathrm{i} \gamma \omega}
\end{gathered}
$$

where $\omega_{p}$ is the plasma frequency, $\omega_{0}$ is the resonance frequency, $\gamma$ is the electron scattering rate, and $F$ is the fractional area of the unit cell occupied by the split ring. The metal gold (Au) and metamaterial layers are sandwiched between the cover which is water buffer with refractive index of 1.332 and high index prism with refractive index of 1.723. By using the Au layer of $d_{2}$ thickness, it is possible to optimize several combinations only for a p-polarized incident light of $632.8 \mathrm{~nm}$ to get sharp resonance curves and minimum reflectivity dips, because the s-polarized light cannot excite electronic surface plasmons [22]. Among these combinations, four-layer and five-layer structures are discussed in this paper.

Table 1 Definition of different structures having different

\begin{tabular}{|c|c|c|}
\hline & $\begin{array}{c}\text { Configuration-1 } \\
\text { (metamaterial layer } \\
\text { based SPR) }\end{array}$ & $\begin{array}{c}\text { Configuration-2 } \\
\text { (dielectric layer based SPR) }\end{array}$ \\
\hline Structure-1 & $\begin{array}{c}\text { Prism-gold } \\
\left(d_{2}\right) \text {-metamaterial } \\
\left(d_{3}\right) \text {-cover }\end{array}$ & $\begin{array}{l}\text { Prism-gold }\left(d_{2}\right) \text {-dielectric } \\
\left(d_{3}\right) \text {-vover }\end{array}$ \\
\hline Structure-2 & $\begin{array}{l}\text { Prism-metamaterial } \\
\left(d_{1}\right) \text {-gold } \\
\left(d_{2}\right) \text {-metamaterial } \\
\left(d_{3}\right) \text {-cover }\end{array}$ & $\begin{array}{l}\text { Prism-dielectric }\left(d_{1}\right) \text {-gold } \\
\left(d_{2}\right) \text {-dielectric }\left(d_{3}\right) \text {-cover }\end{array}$ \\
\hline Structure-3 & $\begin{array}{c}\text { Prism-Gold } \\
\left(d_{2}\right) \text {-Metamaterial }\left(d_{1}+\right. \\
\left.d_{3}\right) \text {-cover }\end{array}$ & $\begin{array}{c}\text { Prism-gold }\left(d_{2}\right) \text {-dielectric }\left(d_{1}+\right. \\
\left.d_{3}\right) \text {-cover }\end{array}$ \\
\hline
\end{tabular}
configurations based on dielectric and metamaterial layers.

Firstly, we solve Maxwell's equations for the four-layer and five-layer SPR sensors and get the Helmholtz equations for p-polarized light, which can be written as

$$
\frac{\partial^{2} H_{y}}{\partial z^{2}}+\left(k_{0}^{2} \varepsilon-\beta^{2}\right) H_{y}=0
$$

where $H_{y}$ represents the magnetic field for $\mathrm{p}$ polarized light, and $\beta$ is the propagation constant in the $x$-direction.

Hence, the solutions of the proposed SPR sensor for different regions can be written as

$$
H_{y}=\left\{\begin{array}{cc}
A c e^{\left(-\mathrm{i} k_{c} z\right)} e^{\mathrm{i}\left(\omega t-k_{x} x\right)} & \text { Water buffer } \\
\left\{A a e^{\left(\mathrm{i} k_{m z} z\right)}+B a e^{\left(-\mathrm{i} k_{m z} z\right)}\right\} e^{\mathrm{i}\left(\omega t-k_{x} x\right)} & \text { Outer MM layer } \\
\left\{A g e^{\left(\mathrm{i} k_{g} z\right)}+B g e^{\left(-\mathrm{i} k_{g} z\right)}\right\} e^{\mathrm{i}\left(\omega t-k_{x} x\right)} & \text { Gold layer } \\
\left\{A m e^{\left(\mathrm{i} k_{m 1} z\right)}+B a e^{\left(-\mathrm{i} k_{m 1} z\right)}\right\} e^{\mathrm{i}\left(\omega t-k_{x} x\right)} \\
\text { Inner MM layer } \\
\left\{A s e^{\left(\mathrm{i} k_{p} z\right)}\right\} e^{\mathrm{i}\left(\omega t-k_{x} x\right)} \quad \text { Prism }
\end{array}\right.
$$


where

$$
\begin{array}{cl}
k_{c}=\sqrt{\beta^{2}-n_{c}^{2} k_{0}^{2}}, & k_{m 2}=\sqrt{\beta^{2}-n_{m 2}^{2} k_{0}^{2}}, \\
k_{g}=\sqrt{n_{g}^{2} k_{0}^{2}-\beta^{2}}, & k_{m 1}=\sqrt{n_{m 1}^{2} k_{0}^{2}-\beta^{2}}, \\
k_{p}=\sqrt{\beta^{2}-n_{p}^{2} k_{0}^{2}} .
\end{array}
$$

Now apply the boundary conditions and consider that the fields and their derivatives should be continuous at the prism-metamaterial, metamaterialgold, gold-metamaterial, and metamaterial-water buffer media boundaries. By matching the field at the boundaries from (4), we get a set of eight equations having eight unknown constants. In order to get field profile for the proposed sensor, the above set of eight equations can be written in the form

$$
\Delta \psi=0
$$

where $\Delta$ is $8 \times 8$ determinant which is obtained from the above set of eight equations.

And

$$
\Psi=\left(A_{c}, A_{m 2}, B_{m 2}, A_{g}, B_{g}, A_{m 1}, A_{m 2}, A_{s}\right) .
$$

The modal field profile of a proposed SPR sensor is also plotted with a given guiding film thickness, and the corresponding effective refractive index $\left(n_{\text {eff }}\right)$ which is calculated from (5) by assuming one of the field amplitudes is equal to one.

For p-polarized incident light, the reflectance $R$ can be predicted from the well-established Fresnel theory based on ray optics [24]. While the detailed derivations are available from many public archives, the resulting Fresnel equations are given as

$$
R=\left|r_{p}\right|^{2}
$$

where

$$
\begin{gathered}
r_{p}=\left|\frac{r_{\mathrm{sm}}+r_{\mathrm{mfac}} e^{2 \mathrm{ik} k_{m} d_{m}}}{1+r_{\mathrm{sm}} r_{\mathrm{mfac}} e^{2 \mathrm{ik} k_{m} d_{m}}}\right|^{2} \\
r_{\mathrm{mfac}}=\frac{r_{\mathrm{mf}}+r_{\mathrm{fac}} e^{2 \mathrm{i} k_{f} d_{f}}}{1+r_{\mathrm{mf}} r_{\mathrm{fac}} e^{2 \mathrm{i} k_{f} d_{f}}}, \quad r_{\mathrm{fac}}=\frac{r_{\mathrm{fa}}+r_{\mathrm{ac}} e^{2 \mathrm{ik} k_{a} d_{a}}}{1+r_{\mathrm{fa}} r_{\mathrm{ac}} e^{2 \mathrm{i} k_{a} d_{a}}}, \\
r_{\mathrm{sm}}=\frac{\left(n_{s}^{2}\right)^{\rho} k_{s}-\left(n_{m}^{2}\right)^{\rho} k_{m}}{\left(n_{s}^{2}\right)^{\rho} k_{s}+\left(n_{m}^{2}\right)^{\rho} k_{m}}, \quad r_{\mathrm{mf}}=\frac{\left(n_{m}^{2}\right)^{\rho} k_{m}-\left(n_{f}^{2}\right)^{\rho} k_{f}}{\left(n_{m}^{2}\right)^{\rho} k_{m}+\left(n_{f}^{2}\right)^{\rho} k_{f}},
\end{gathered}
$$

$$
r_{\mathrm{fa}}=\frac{\left(n_{f}^{2}\right)^{\rho} k_{f}-\left(n_{a}^{2}\right)^{\rho} k_{a}}{\left(n_{f}^{2}\right)^{\rho} k_{f}+\left(n_{a}^{2}\right)^{\rho} k_{a}}, \quad r_{\mathrm{ac}}=\frac{\left(n_{a}^{2}\right)^{\rho} k_{a}-\left(n_{c}^{2}\right)^{\rho} k_{c}}{\left(n_{a}^{2}\right)^{\rho} k_{a}+\left(n_{c}^{2}\right)^{\rho} k_{c}}
$$

where $\rho$ is the polarization index that equals to 1 for p-polarized light.

The SPR reflectance can be readily determined as a function of the pertinent variables by numerically solving the above Fresnel equations. The final result is a normalized reflection term which represents the amount of light reflected from a surface. It is obvious that the model can be used to predict the SPR response with an angle modulated SPR sensor design.

\section{Numerical results and discussion}

This paper deals with optical SPR sensors by making use of isotropic and homogeneous metamaterials. We have chosen the following parameters, an operating wavelength $\lambda_{0}=632.8 \mathrm{~nm}$ and $d_{1}=300 \mathrm{~nm}, d_{2}=35 \mathrm{~nm}$, and $d_{3}=300 \mathrm{~nm}$. Values of negative dielectric and magnetic constants for metamaterial layers are $\left\{\varepsilon_{m}, \mu_{m}\right\}=$ $\{-0.66-0.001 \mathrm{i},-2\}$, and these sets generate a negative refractive index of $n=-(\varepsilon \mu)^{1 / 2}$ $[19,20]$. Refractive indices for the prism, $\mathrm{Au}$, dielectric layer, and water buffer (cover) are $1.723,0.2184+3.5113 \mathrm{i}, 2.198$, and 1.332, respectively [22].

Now we plot the reflectance curve for the proposed SPR sensor using a very simple approach, i. e. Fresnel's equations, compute the reflectance for various values of the incident angle $(\theta)$, and plot the curves between the reflectivity and incident angle for the four-layer and five-layer structures as illustrated in Figs. 2, 3, and 4. These curves show SPR sensors with the metamaterial layer providing the minimum reflectance that can be seen from Table2. 


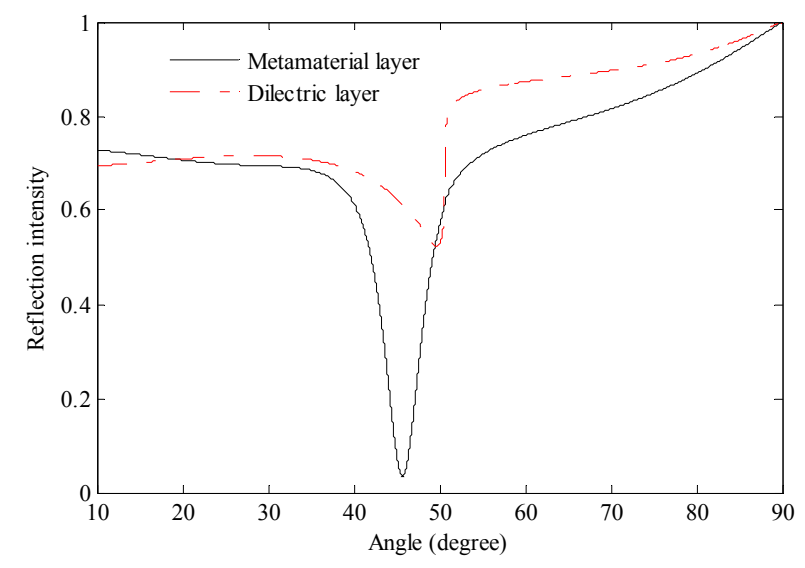

Fig. 2 Calculated reflectance curve for the dielectric layer (dashed line) and metamaterial based (solid line) SPR sensor, i.e. Structure- 1 as a function of incident angle for $d_{2}=35 \mathrm{~nm}, d_{3}=$ $300 \mathrm{~nm}$ : refractive indices for the prism, Au metal, dielectric layer, and water buffer are $1.723,0.218+3.5113 \mathrm{i}, 2.198$, and 1.332, respectively; refractive index value of $\left\{\varepsilon_{m}, \mu_{m}\right\}$ of the metamaterial is $\{-0.66-0.001 \mathrm{i},-2\}$ for the four-layer structure.

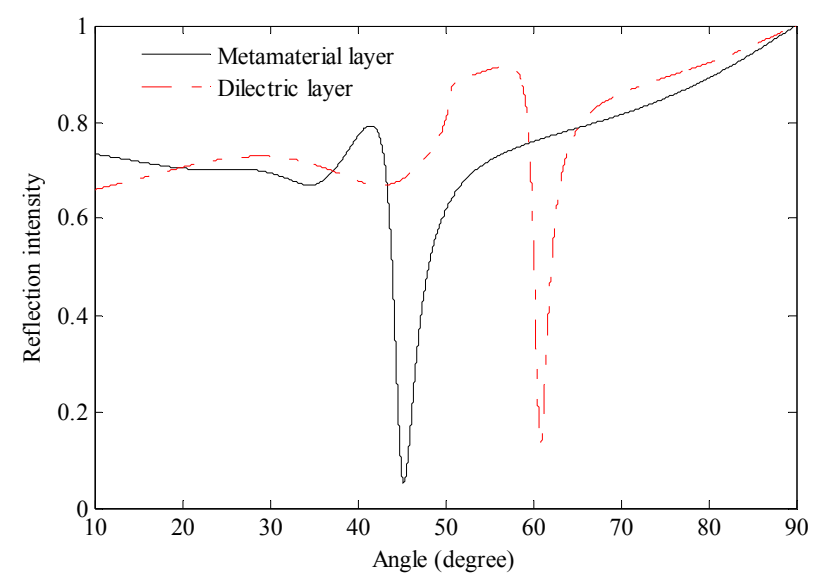

Fig. 4 Calculated reflectance curve for the dielectric layer (dashed line) and metamaterial based (solid line) SPR sensor i.e. Structure- 3 as a function of the incident angle for $d_{2}=35 \mathrm{~nm}$ and $d=d_{1}+d_{3}=600 \mathrm{~nm}$ : refractive indices for the prism, Au metal, dielectric layer, and water buffer are $1.723,0.218+3.5113 \mathrm{i}$, 2.198 , and 1.332, respectively; refractive index values of the metamaterial is $\{-0.66-0.001 i,-2\}$ for the five-layer structure.

Table 2 Values of Reflectance for SPR layered structures.

\begin{tabular}{ccccc}
\hline \multirow{2}{*}{ Structure } & \multicolumn{2}{c}{$\begin{array}{c}\text { Configuration-1 } \\
\text { (metamaterial layer based SPR) }\end{array}$} & \multicolumn{2}{c}{$\begin{array}{c}\text { Configuration-2 } \\
\text { (dielectric layer based SPR) }\end{array}$} \\
\cline { 2 - 5 } & $\begin{array}{c}\text { Incident } \\
\text { angle }\end{array}$ & $\begin{array}{c}\text { Reflectance } \\
\text { value }\end{array}$ & $\begin{array}{c}\text { Incident } \\
\text { angle }\end{array}$ & $\begin{array}{c}\text { Reflectance } \\
\text { value }\end{array}$ \\
\hline Structure-1 & 45.6962 & 0.03544 & 49.755 & 0.52346 \\
Structure-2 & 42.2682 & 0.00009 & 49.7814 & 0.53153 \\
Structure-3 & 45.3072 & 0.05312 & 60.9002 & 0.13134 \\
\hline
\end{tabular}

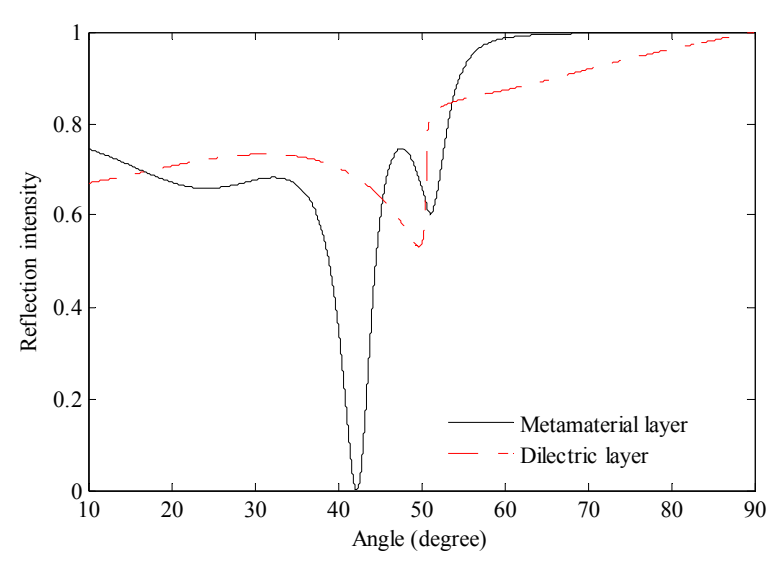

Fig. 3 Calculated reflectance curve for the dielectric layer (dashed line) and metamaterial based (solid line) SPR sensor, i.e. Structure- 2 as a function of the incident angle for $d_{1}=300 \mathrm{~nm}$, $d_{2}=35 \mathrm{~nm}$ and $d_{3}=300 \mathrm{~nm}$ : refractive indices of the prism, $\mathrm{Au}$ metal, dielectric layer, and water buffer are 1.723, $0.218+3.5113 \mathrm{i}, 2.198$, and 1.332 , respectively; refractive index value of the metamaterial is $\{-0.66-0.001 \mathrm{i},-2\}$ for the five-layer structure.

It is seen from Table 2 and Fig. 3 that the bi-metamaterial based SPR sensor provides the sharp resonance curve and minimum reflectivity dip, and increases the dynamic range in comparison with those proposed by K. S. Lee et al. [21] and Y. K. Prajapati et al. [22]. K. S. Lee et al. obtained the incident angle and reflectance value as $51.05799^{\circ}$ and 0.00995 for the bi-metallic five-layer optical SPR sensor, respectively.

Prajapati et al. obtained the incident angle and reflectance value as $50.63343^{\circ}$ and 0.00317 using the metal and bi-metamaterial layer of the five-layer optical SPR sensor, respectively. When the dip is sharper, the reading is more accurate. Moreover, the choice of the bi-metamaterial layer plays a significant role in the reflectance dip sharpness.

Our computed results are better in terms of the incident angle and reflectance value of the respective optical SPR sensor using the bi-metamaterial layer in place of dielectric materials as shown in Table 2.

It is observed from Fig. 2, that for the four-layer metamaterial based SPR sensor, we can obtain the incident angle and reflectance dip value as $45.6962^{\circ}$ and 0.03544 , respectively. While in Fig. 4 , the 
five-layer metamaterial based SPR sensor structure can be converted into the four-layer metamaterial based SPR sensor structure by merging the two consecutive metamaterial layers above the gold layer, and we obtain the incident angle and reflectance dip value as $45.3072^{\circ}$ and 0.05312 , respectively.

It means that the incident angle of the five-layer metamaterial sensor, i.e. Structure-3 when converted in the four-layer metamaterial sensor by merging two consecutive metamaterial layers, is slightly reduced with respect to the four-layer metamaterial sensor, i.e. Structure-1 but the reflectance value slightly increases as shown in Table 2. It is also seen from Table 2 that when the sensor structure is changed from the four layer to the five layer, i.e. from Structure -1 and Structure- 3 to Structure-2, the reflectance approaches to the minimum and incident angle decreases.

It is seen that the thickness of the metal layer plays a significant role in the sharpness and depth of the reflectance dip.

The calculated reflectance is shown in Fig. 5 for three different thicknesses of the metal layer. At a metal thickness of $30 \mathrm{~nm}$, the reflectance approaches zero, which is defined as the optimum metal

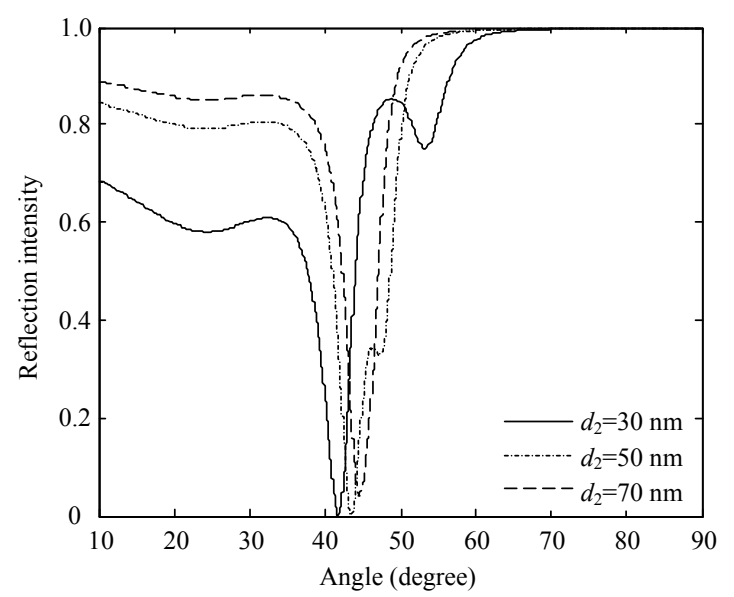

Fig. 5 Calculated reflectance curve for a metamaterial based SPR sensor, i.e. Structure-2 as a function of the incident angle for different values of the gold metal layer thickness for $d_{1}=$ $300 \mathrm{~nm}, d_{3}=300 \mathrm{~nm}$ : refractive indices for the prism, Au metal, and water buffer are 1.723, 0.218+3.5113i, and 1.332, respectively; refractive index values of the metamaterial is $\{-0.66-0.001 i,-2\}$ for the five-layer structure. thickness for the proposed SPR sensors.

The thickness of the metamaterial layer has a big influence on the angular position of the incident angle as shown in Fig. 6.

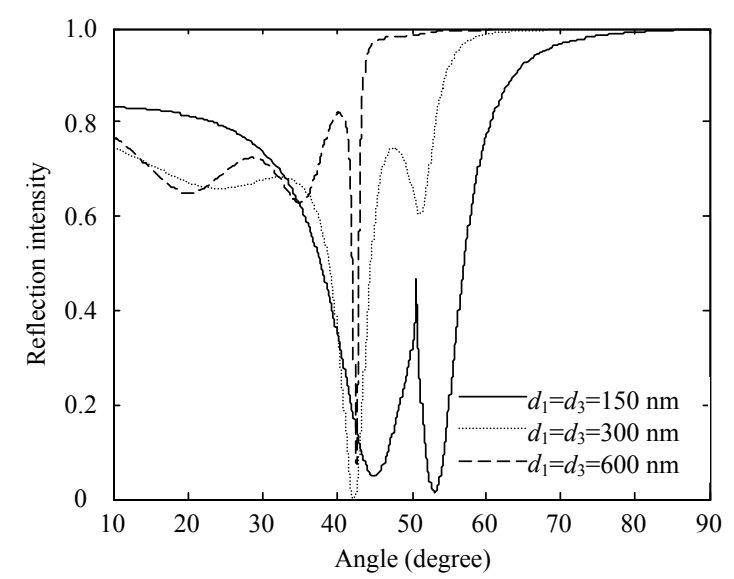

Fig. 6 Calculated reflectance curve for a metamaterial based SPR sensor, i.e. Structure-2 as a function of the incident angle for different values of the metamaterial layer thickness for $d_{2}=35 \mathrm{~nm}$; refractive indices for the prism, Au metal, and water buffer are $1.723,0.218+3.5113 \mathrm{i}$, and 1.332 , respectively; refractive index values of the metamaterial is $\{-0.66-0.001 i,-2\}$ for the five-layer structure.

In Fig. 6, on decreasing the thickness of both metamaterial layers $d_{1}=d_{3}=150 \mathrm{~nm}$, the incident angle and reflectance values are $53.123^{\circ}$ and 0.01388 , and on increasing the thickness of both metamaterial layers $d_{1}=d_{3}=600 \mathrm{~nm}$, the incident angle and reflectance values are $42.577^{\circ}$ and 0.07314, respectively. At more than $300 \mathrm{~nm}$ thickness of metamaterial layers, there is a slightly increase in the incident angle and reflectance value but not much. This means on increasing the thickness of metamaterial layers, the incident angle and reflectance values are almost constant due to the penetration depth of the evanescent field.

At less than $300 \mathrm{~nm}$ thickness of metamaterial layers, there is an increase in the incident angle and reflectance value, i. e. $53.123^{\circ}$ and 0.01388 as shown in Fig. 6, respectively. Therefore, at a metamaterial thickness of $300 \mathrm{~nm}$, the reflectance approaches to zero, which is defined as the optimum metamaterial thickness for the proposed SPR sensors.

Also, we investigate the effect of the electric 
permittivity $\left(\varepsilon_{m}\right)$ and magnetic permeability $\left(\mu_{m}\right)$ of metamaterial layers on the reflectance curves. As the absolute values of $\varepsilon_{m}$ and $\mu_{m}$ increase, the reflectance dip becomes sharper, and the incident angle is obtained at a higher value, as shown in Figs. 7 and 8 .

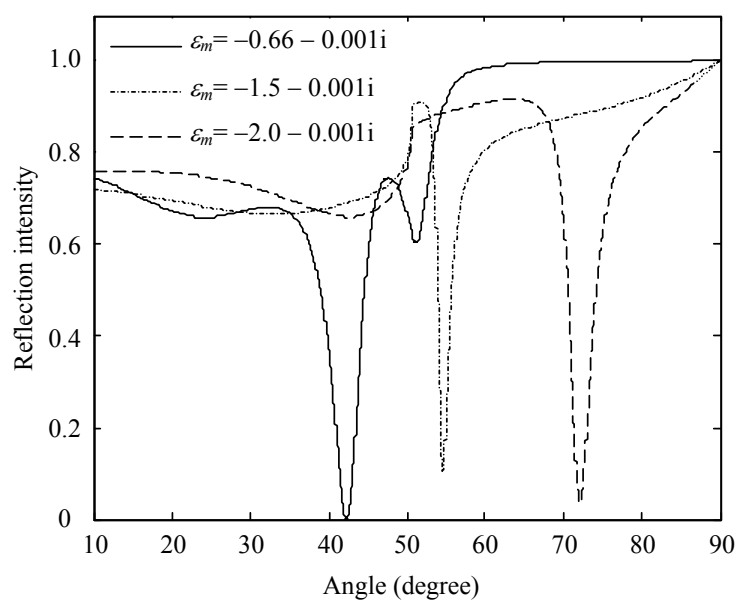

Fig. 7 Calculated reflectance curve for a metamaterial based SPR sensor, i.e. Structure-2 as a function of the incident angle for different values of the permittivity of the metamaterial layer for $d_{1}=300 \mathrm{~nm}, d_{2}=35 \mathrm{~nm}$, and $d_{3}=300 \mathrm{~nm}$ : refractive indices for the prism, Au metal, and water buffer are 1.723, 0.218+3.5113i, and 1.332, respectively; refractive index values of $\left\{\mu_{m}\right\}$ for the metamaterial is $\{-2\}$ for the five-layer structure.

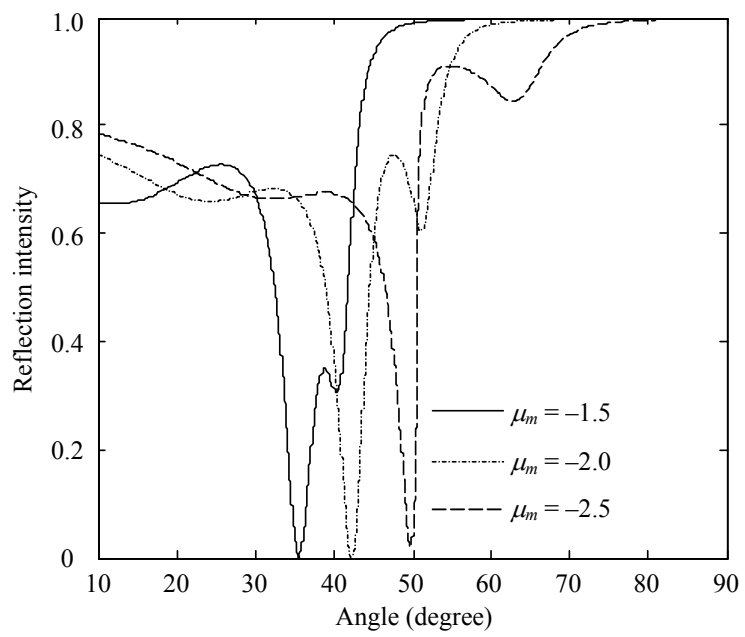

Fig. 8 Calculated reflectance curve for a metamaterial based SPR sensor, i.e. Structure- 2 as a function of the incident angle for different values of the permittivity of the metamaterial layer for $d_{1}=300 \mathrm{~nm}, d_{2}=35 \mathrm{~nm}$, and $d_{3}=300 \mathrm{~nm}$ : refractive indices for the prism, Au metal, and water buffer are 1.723, 0.218+3.5113i, and 1.332, respectively; refractive index values of $\left\{\varepsilon_{m}\right\}$ for the metamaterial is $\{-0.66-0.001 i\}$ for the five-layer structure.
Now plot the model field distribution of the proposed five-layer metamaterial SPR sensor using a very simple approach as described above in Section 2. The field distribution obtained by this approach is shown in Fig. 9 for p-polarized light.

The field distribution is in the expected shape. The analysis of Fig. 9 results in the conclusion that the presence of the gold layer corresponds to an improved field at the metamaterial and gold layer boundary.

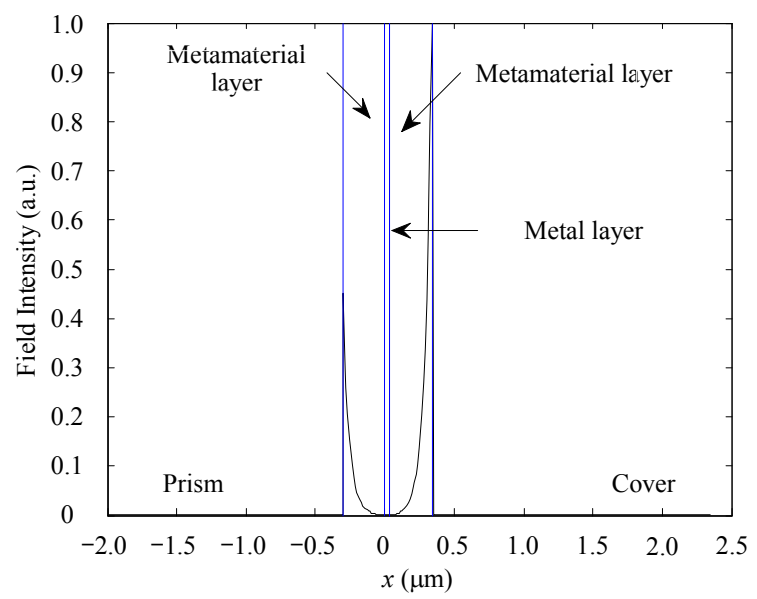

Fig. 9 Normalized modal field distribution of a five-layer metamaterial optical SPR sensor, i.e. Structure-2 for p-polarized light.

Finally, the possibility of finding a physical system that has a negative index at the desired wavelength should be pointed out. At present, extending the resonance frequency band of the metamaterial from low to high, even to the visible light band, is an important research direction.

\section{Conclusions}

The optical SPR sensor with bi-metamaterial layer properties can be optimized in terms of achieving the largest shift in the incident angle. A much sharper reflectance dip is obtained in the presence of a metal layer between metamaterial layers. The use of metamaterial layers is confirmed to have an advantage in increasing the reflectance dip of the optical SPR sensor, thus improving the sensitivity in term of the sensing resolution and 
dynamic range. This is advantageous for sensing applications. The proposed structure is found to have an advantage over the conventional optical SPR as well as the bi-metallic optical SPR. The validity of the magnetic field results is proved on the basis of smooth match of the fields in the different layers of the proposed optical SPR sensor.

\section{Acknowledgment}

The present work is partially supported by the Department of Science and Technology (DST), New Delhi, India under the fast track young scientist Scheme No. SB/FTP/ETA-0478/2012.

Open Access This article is distributed under the terms of the Creative Commons Attribution License which permits any use, distribution, and reproduction in any medium, provided the original author(s) and source are credited.

\section{References}

[1] J. J. Burke, G. I. Stegeman, and T. Tamir, "Surface polariton like waves guided by thin lossy metalfilms," Physical Review B, 1986, 33(8): 5186-5201.

[2] H. Raether, Surface plasmons on smooth and rough surfaces and on grating. Berlin, Germany: Springer-Verlag, 1988.

[3] S. A. Zynio, A. V. Samoylov, E. R. Surovtseva, V. M. Mirsky, and Y. M. Shirshov, "Bimetallic layers increase sensitivity of affinity sensors based on SPR," Sensors, 2002, 2(2): 62-70.

[4] J. Homola, "Present and future of Surface plasmon resonance biosensors," Analytical and Bioanalytical Chemistry, 2003, 377(3): 528-539.

[5] E. Kretschmann and H. Raether, "Radiative decay of non radiative surface plasmons excited by light," Zeitschrift für Naturforschung A, 1968, 23(12): 2135-2136.

[6] J. Dostálek, A. Kasry, and W. Knoll, "Long range surface plasmons for observation of biomolecular binding events at metallic surfaces," Plasmonics, 2007, 2(3): 97-106.

[7] G. G. Nenninger, P. Tobiška, J. Homola, and S. S. Yee, "Long-range surface plasmons for high-resolution SPR sensors," Sensors and Actuators B: Chemical, 2001, 74(1-3): 145-151.
[8] B. H. Ong, X. Yuan, S. C. Tjin, J. Zhang, and H. M. $\mathrm{Ng}$, "Optimised film thickness for maximum evanescent field enhancement of a bimetallic film SPR biosensor," Sensors and Actuators B: Chemical, 2006, 114(2): 1028-1034.

[9] F. C. Chien and S. J. Chen, "A sensitivity comparison of optical biosensor based on four different SPR modes," Biosensors and Bioelectronics, 2004, 20(3): 633-642.

[10] J. Homola, Surface Plasmon Resonance Based Sensors. Berlin, Germany: Springer-Verlag, 2006.

[11] X. D. Hoa, A. G. Kirk, and M. Tabrizian, "Towards integrated and sensitive surface plasmon resonance biosensors: a review of recent progress," Biosensors and Bioelectronics, 2007, 23(3): 151-160.

[12] C. W. Lin, K. P. Chen, C. N Hsiao, S. Lin, and C. K. Lee, "Design and fabrication of an alternating dielectric multi-layer device for surface plasmon resonance sensor," Sensors and Actuators B: Chemical, 2006, 113(1): 169-176.

[13] V. Veselago, "The electrodynamics of substance with simultaneously negative values of $\varepsilon$ and $\mu$," Soviet Physics Uspekhi, 1968, 10(9): 509-514.

[14] D. Sharma, A. Verma, Y. K. Prajapati, V. Singh, and J. P. Saini, "Forward and backward wave propagation in multilayer planar waveguide using metamaterials layer," Optical and Quantum Electronics, 2013, 45(2): 105-114.

[15] J. B. Pendry, A. J. Holden, D. J. Robbins, and W. J. Stewart, "Magnetism from conductors and enhanced nonlinear phenomena," IEEE Transactions on Microwave Theory and Techniques, 1999, 47(11): 2075-2084.

[16] R. A. Shelby, D. R. Smith, and S. Schultz, "Experimental verification of a negative index of refraction," Science, 2011, 292(5514): 77-79.

[17] J. B. Pendry, "Negative refraction makes a perfect lens," Physical Review Letters, 2000, 85(18): 3966-3969.

[18] T. Chen, S. Li, and H. Sun, "Metamaterials application in sensing," Sensors, 2012, 12(3): 2742-2765.

[19] A. Upadhyay, Y. K. Prajapati, V. Singh, and J. P. Saini, "Sensitivity estimation of metamaterial loaded planar waveguide sensor," Optical and Quantum Electronics, 2014, 46(12): 1.

[20] A. Upadhyay, Y. K. Prajapati, V. Singh, and J. P. Saini, "Comprehensive study of reverse index waveguide based sensor with metamaterial as a guiding layer," Optics Communications, 2015, 
348: 71-76.

[21] K. S. Lee, J. M. Son, D. Y. Jeong, T. S. Lee, and W. M. Kim, "Resolution enhancement in SPR sensor based on waveguide coupled mode by combining a bimetallic approach," Sensors, 2010, 10(12): 11390-11399.

[22] Y. K. Prajapati, A. Yadav, A. Verma, V. Singh, and J. P. Saini, "Effect of metamaterial layer on optical surface plasmon resonance sensor," Optik -
International Journal for Light and Electron Optics, 2013, 124(18): 3607-3610.

[23] K. Park, B. J. Lee, C. Fu, and Z. M. Zhang, "Study of the surface and bulk polaritons with negative index metamaterial," Journal of the Optical Society of America B, 2005, 22(5): 1016-1023.

[24] M. Born and E. Wolf, Principles of optics. Oxford, Britain: Cambridge University Press, 1980. 